\title{
Synaptic symmetry breaking by spike timing dependent synaptic
} plasticity

\section{Chang-Woo Shin* and Seunghwan Kim}

\author{
Address: Asia Pacific Center for Theoretical Physics and Nonlinear and Complex Systems Laboratory, Department of Physics, Pohang University \\ of Science and Technology, San 31, Hyoja-dong, Nam-gu, Pohang, Gyeongbuk, Korea, 790-784 \\ Email: Chang-Woo Shin* - shine@postech.ac.kr \\ * Corresponding author
}

from Seventeenth Annual Computational Neuroscience Meeting: CNS*2008

Portland, OR, USA. 19-24 July 2008

Published: II July 2008

BMC Neuroscience 2008, 9(SuppI I):PI00 doi:I0.1 I86/I47I-2202-9-SI-PI00

This abstract is available from: http://www.biomedcentral.com/I47I-2202/9/SI/PI00

(c) 2008 Shin and Kim; licensee BioMed Central Ltd.

Recent experimental studies have shown that in various brain regions, such as the hippocampus and the neocortex, both the sign and the magnitude of synaptic modification depend on the precise temporal relation of spike timing of two neurons, which is called spike timing dependent synaptic plasticity (STDP). Due to the asymmetry of the critical window of STDP, STDP tends to lead morphologically reciprocal synaptic connections between two neurons into a functionally uni-directional synaptic connection. We study the spontaneous symmetry breaking of bi-directional synaptic connections between two neurons, which leads the synaptic competition in a larger network. We show that STDP is optimized to the symmetry breaking for the experimentally observed time scale range of the critical window. 